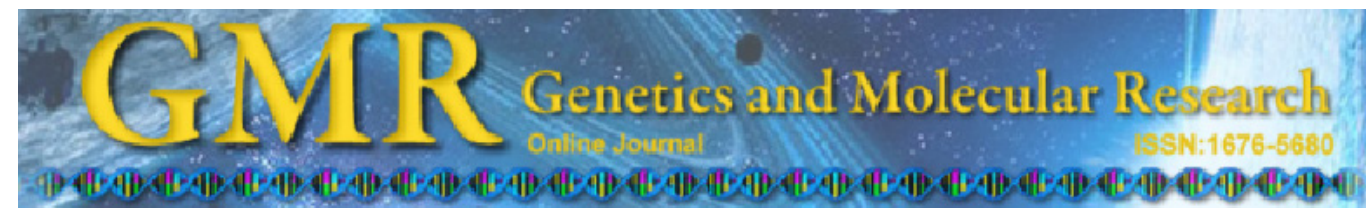

Short Communication

\title{
Genome-wide identification and characterization of simple sequence repeat loci in grape phylloxera, Daktulosphaira vitifoliae
}

\author{
H. Lin ${ }^{1}$, M.S. Islam ${ }^{1,2}$ and D.W. Ramming ${ }^{1}$ \\ ${ }^{1}$ Crop Diseases, Pests and Genetics Research Unit, USDA-ARS, Parlier, CA, USA \\ ${ }^{2}$ Department of Viticulture and Enology, University of California Davis, \\ Davis, CA, USA \\ Corresponding author: H. Lin \\ E-mail: Hong.Lin@ars.usda.gov
}

Genet. Mol. Res. 11 (2): 1409-1416 (2012)

Received October 6, 2011

Accepted February 27, 2012

Published May 15, 2012

DOI http://dx.doi.org/10.4238/2012.May.15.11

\begin{abstract}
A genome-wide sequence search was conducted to identify simple sequence repeat (SSR) loci in phylloxera, Daktulosphaira vitifoliae, a major grape pest throughout the world. Collectively, 1524 SSR loci containing mono-, di-, tri-, tetra-, penta-, and hexanucleotide motifs were identified. Among them, trinucleotide repeats were the most abundant in the phylloxera genome (34.4\%), followed by hexanucleotide $(20.4 \%)$ and dinucleotide (19.6\%) repeats. Mono-, tetra- and pentanucleotide repeats were found at a frequency of 1.3, 11.2 and $12.9 \%$, respectively. The abundance and inherent variations in SSRs provide valuable information for developing molecular markers. The high levels of allelic variation and codominant features of SSRs make this marker system a useful tool for genotyping, diversity assessment and population genetic studies of reproductive characteristics of phylloxera in agricultural and natural populations.
\end{abstract}

Key words: Grape phylloxera; Simple sequence repeat marker; Genetic diversity; Population genetics 


\section{INTRODUCTION}

Grape phylloxera, Daktulosphaira vitifoliae Fitch (Homoptera: Phylloxeridae), is a viticultural pest specialized in feeding on grapevine (Vitis spp). Phylloxera has been destroying vineyards around the world for the past 140 years (Granett et al., 1996). It is found throughout the Americas where it appears to have coevolved with the endemic Vitis spp (Wapshere and Helm, 1987). This pest was accidentally introduced to European viticultural regions from North America in the mid 1800s. By 1900, two-thirds of all Vitis vinifera vineyards in Europe had been destroyed. Since then, phylloxera has spread to most grape-growing areas of the world, including New Zealand, Australia, South Africa, South America, and Canada (Skinkis et al., 1995). It is regarded as one of the most important viticultural pests in the world (Granett et al., 1996).

Although resistant rootstocks have effectively defended vineyards against this pest, the durability of resistant grape plants depends on the variability and adaptability of pest populations rather than the resistance gene itself. In California, for example, the emergence of more aggressive strains of phylloxera, called biotype B, overcame the limited resistance of the AXR\#1 rootstock (Granett et al., 2001). Damage intensity caused by plant pests most likely correlates with diversity and population size. The insect varies genetically relative to its performance on hosts. The use of insecticides has limited effects on controlling the population, and other control methods are unproven. Therefore, information regarding the genetic diversity, population structure, and reproductive characteristics of grape phylloxera within and among various grape rootstocks is important for understanding host selection and adaptation and designing appropriate pest management strategies.

Simple sequence repeat (SSR markers), also known as microsatellite makers or variable number tandem repeats, are considered a powerful tool for distinguishing genetic diversity, studying populations, and determining reproductive characteristics in various organisms. The high level of polymorphism, easy adaption of high throughput capability, extensive genome coverage, and small amount of DNA required for analysis make this marker system favorable over other genetic markers. SSR makers have been isolated from grape phylloxera in previous studies. Corrie et al. (2002) initially isolated four SSR makers. Lin et al. (2006) also isolated 19 SSRs, but only seven were polymorphic across sample populations in California and Europe. In this study, we identified and developed a large set of new SSR markers for grape phylloxera.

\section{MATERIAL AND METHODS}

Phylloxera samples were collected from own-rooted Chardonnay (defined as biotype A) at the University of California (Davis, CA, USA) vineyard and AxR\#1 rootstock (defined as biotype B) in Mendocino County, California. Genomic DNA was isolated following a procedure described elsewhere (Lin and Walker, 1996). DNA quality was evaluated using 1.2\% agarose gel. DNA samples were then quantified using a fluorometer and the PicoGreen method. The phylloxera genome DNA samples were then used for 454 pyrosequencing with Titanium kit by Roche GS-FLX Sequencer (Roche, Branford, CT, USA) according to manufacturer instructions. Sequencing data were de novo assembled with Newbler version 2.0 (Roche).

To identify putative microsatellite regions in the phylloxera genome, we used the Tandem Repeats Finder software (Benson, 1999). After the identification of various motif 
repeat regions, sequences that flanked the prospective repeat motifs of $200 \mathrm{bp}$ upstream and downstream were extracted from the output file. Standalone BLASTn analyses were performed to compare sequences derived from biotype A and biotype B phylloxera. SSR-containing sequences were separated into three groups: 1) SSR sequences present in biotypes A, 2) SSR sequences present in biotypes B, and 3) SSR sequences present in both biotypes. After removing redundant sequences from each group, we identified more than a thousand SSR loci with various repeat motifs. Prospective SSR primers were designed from the identified loci using the Molecular Beacon Designer software (version 7.0) with the following criteria: $35-55 \% \mathrm{GC}$ content, $50^{\circ}-62^{\circ} \mathrm{C}$ melting temperature, and 132-290-bp amplification products containing repeat units $\geq 6$.

\section{RESULTS AND DISCUSSION}

In total 1524 SSR loci with various repeat motifs were identified. Among them, 21 (1.3\%), 299 (19.6\%), 524 (34.4\%), 172 (11.2\%), 197 (12.9\%), and 311 (20.4\%) were observed as mono-, di-, tri-, tetra, penta-, and hexanucleotide repeats, respectively (Figure 1).

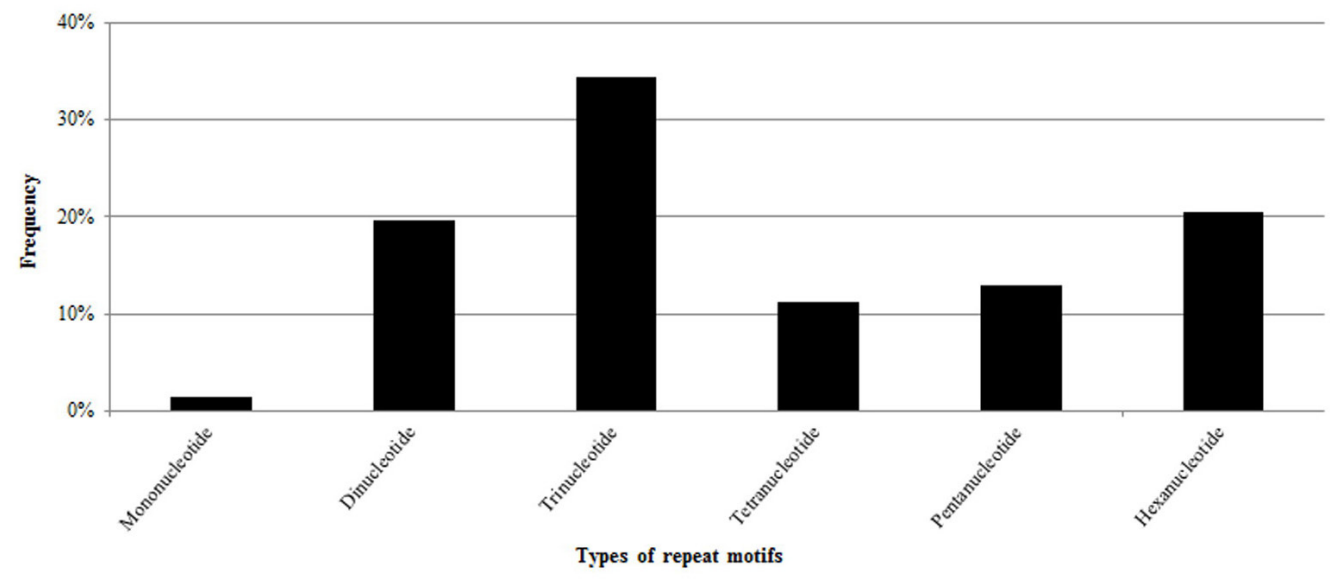

Figure 1. Types of simple sequence repeat frequencies throughout the genome of grape phylloxera (Daktulosphaira vitifoliae).

From these various SSR repeat regions, a total of 112 SSR primer pairs were tentatively designed (Table 1). Among them, 49 and 48 primer pairs were designed from the sequences obtained from biotype A and biotype B, respectively. In addition, 15 SSR primer pairs that detect sequence polymorphism between biotype A and biotype B were also designed (see Table 1).

Combining a next-generation deep-sequencing strategy with an in silico mining approach provided an effective way to identify SSR loci in the phylloxera genome. The new set of markers enhances the ability to characterize population structure, reproduction mode, and adaptation of grape phylloxera to various rootstocks in grape-growing regions around the world. 
Table 1. Descriptions of 112 simple sequence repeat (SSR) makers developed form the genome-wide sequence search of grape phylloxera (Daktulosphaira vitifoliae).

\begin{tabular}{|c|c|c|c|c|c|}
\hline Locus & Primer sequences $\left(5^{\prime}-3^{\prime}\right)$ & Repeats & Fragment size & $\mathrm{Ta}\left({ }^{\circ} \mathrm{C}\right)$ & $\begin{array}{c}\text { GenBank } \\
\text { accession No }\end{array}$ \\
\hline \multicolumn{6}{|c|}{49 SSR markers developed from biotype A } \\
\hline $\begin{array}{l}\text { DVSSR_A001-F } \\
\text { DVSSR_A001-R }\end{array}$ & $\begin{array}{l}\text { CAGTGATATTTGTCGGAGAGG } \\
\text { CCGGTCATTGGTTGTTATGG }\end{array}$ & $(\mathrm{CA})_{12} \mathrm{TA}(\mathrm{CA})_{6}$ & 166 & 53 & GF111391 \\
\hline $\begin{array}{l}\text { DVSSR_A002-F }^{-} \text {A002-R } \\
\text { DVSSR A002-R }\end{array}$ & $\begin{array}{l}\text { CGGCGAACACGTATAATG } \\
\text { AACGGCATAAGCAATAAGC }\end{array}$ & $(\mathrm{GT})_{15}$ & 250 & 50 & GF111392 \\
\hline DVSSR_A003-F & GCGCTGGCCGAGAGCAAGAG & $(\mathrm{AT})_{11} \mathrm{TTATGT}(\mathrm{AT})_{4}$ & 221 & 63 & GF111393 \\
\hline DVSSR_A003-R & CGGCGGCGAACGCTCTATCTTG & & & & \\
\hline $\begin{array}{l}\text { DVSSR_A004-F } \\
\text { DVSSR A004-R }\end{array}$ & $\begin{array}{l}\text { CGGTGCCAGATTGCTTATGAAC } \\
\text { AGTCAATCGAGATAAGCTGAAAGAG }\end{array}$ & $\left.(\mathrm{TA})_{10} \mathrm{TGTAC} \mathrm{TA}\right)_{3}$ & 234 & 57 & GF111394 \\
\hline $\begin{array}{l}\text { DVSSR_A005-F } \\
\text { DVSSR A005-R }\end{array}$ & $\begin{array}{l}\text { CACTTAGCTTCCTTTCTATACTTGG } \\
\text { CAGCATGTCACTAGGGATTGG }\end{array}$ & $\operatorname{ATAC}(\mathrm{AT})_{10} \mathrm{GT}(\mathrm{AT})_{3}$ & 139 & 55 & GF111395 \\
\hline DVSSR_A006-F & ATCCTCACTCTTCCTCTTTCTG & $\begin{array}{c}(\mathrm{ATT})_{12} \mathrm{GT}(\mathrm{AC})_{4} \mathrm{AT} \\
(\mathrm{AC})_{5} \mathrm{C}(\mathrm{AT})_{13}\end{array}$ & 236 & 55 & GF111396 \\
\hline DVSSR_A006-R & CACGGCGTAGTGGATATGC & & & & \\
\hline $\begin{array}{l}\text { DVSSR_A007-F } \\
\text { DVSSR_A007-R }\end{array}$ & $\begin{array}{l}\text { ATAGGGATAAGGAAACGATGGG } \\
\text { GAGGCGATAGCAGAGTATGG }\end{array}$ & $(\mathrm{AT})_{12} \mathrm{~T}(\mathrm{AT})_{5}$ & 214 & 55 & GF111397 \\
\hline DVSSR_A008-F & TGCGCTGGACTTAGTGTTAC & $\mathrm{ATCT}(\mathrm{AT})_{3} \mathrm{ACT}(\mathrm{AT})_{12}$ & 132 & 54 & GF111398 \\
\hline $\begin{array}{l}\text { DVSSR_A008-R } \\
\text { DVSSR_A009-F }\end{array}$ & $\begin{array}{l}\text { TATCCACTGTTTACGGTTGAAC } \\
\text { CGGCAAGCAGCATCAAGC }\end{array}$ & & & & \\
\hline DVSSR_A009-R & TTCCAGGTGTGTGTATGTGTTG & C) ${ }_{1}$ & 190 & 57 & GF111399 \\
\hline $\begin{array}{l}\text { DVSSR_A010-F } \\
\text { DVSSR_A010-R }\end{array}$ & $\begin{array}{l}\text { TACTCTTAAAAGAAGCATAACATAGG } \\
\text { ATAATTTGCATGGTGGAAGTTTAG }\end{array}$ & $\operatorname{TAAA}(\mathrm{TA})_{11} \ldots(\mathrm{TA})_{3}$ & 184 & 54 & GF111400 \\
\hline DVSSR_A011-F & TTATTGCCGTCGGAGGATCG & $(\mathrm{TG})_{13} \mathrm{TAC}(\mathrm{TG})_{2}$ & 206 & 57 & GF111401 \\
\hline $\begin{array}{l}\text { DVSSR_A011-R } \\
\text { DVSSR_A012-F }\end{array}$ & $\begin{array}{l}\text { TGGATTGTGGCGGTGATGG } \\
\text { AAGGCATTAACTTGTCGCATTC }\end{array}$ & $\begin{array}{c}(\mathrm{CA})_{3} . . .(\mathrm{CA})_{15} \mathrm{TA}(\mathrm{CA})_{2} \\
\mathrm{TA}(\mathrm{CA})_{7}\end{array}$ & 223 & 56 & GF111402 \\
\hline DVSSR_A012-R & GTAGCATGTGGACTTGACTGG & & & & \\
\hline DVSSR_A013-F & GCTTTCACCAACTACCGTACC & $(\mathrm{AC})_{12}$ & 146 & 56 & GF111403 \\
\hline DVSSR_A013-R & ТСССТСАТАСАСТСАСАCTCG & & & & \\
\hline DVSSR_A014-F & TGGTCCTGGTGGCTTTGG & $(\mathrm{AT})_{11} \mathrm{GT}(\mathrm{AT})_{2}$ & 132 & 56 & GF111404 \\
\hline DVSSR_A014-R & TCCACTGCCTCGATCTTGC & & & & \\
\hline DVSSR_A015-F & ACACGCTATATATGATGGTTGG & $(\mathrm{AT})_{3} . .(\mathrm{AT})_{11}$ & 221 & 54 & GF111405 \\
\hline DVSSR_A015-R & CACGTTTAGTACAACAGACCTC & & & & \\
\hline DVSSR_A016-F & TTGTCAGTTAGGTCTGAGATAC & $(\mathrm{TA})_{5} \ldots(\mathrm{TA})_{9}$ & 183 & 52 & GF111406 \\
\hline DVSSR_A016-R & CAACCATCTTAATCTTCCTACC & & & & \\
\hline DVSSR_A017-F & ACAGTTAGCAGATGATTGGAAC & $(\mathrm{TAT})_{6} \mathrm{AT}(\mathrm{TAT})_{3}$ & 159 & 54 & GF111407 \\
\hline DVSSR_A017-R & CACAAGCATCTTCAGATAGGC & & & & \\
\hline $\begin{array}{l}\text { DVSSR_A018-F } \\
\text { DVSSR_A018-R }\end{array}$ & TATGATCGTCACAGAGGAAACC & $(\mathrm{ATT})_{11}$ & 230 & 55 & GF111408 \\
\hline DVSSR_A018-R & ATCTTTCGCCAATGTTCAAGTG & & & & \\
\hline DVSSR_A019-F & GGCAGTGACCCATGACAG & $(\mathrm{TAT})_{8}$ & 177 & 54 & GF111409 \\
\hline DVSSR_A019-R & GGATACGGTACACAGAAAACG & & & & \\
\hline DVSSR_A020-F & CTCTAGGACACTCATGATTGC & $(\mathrm{ATA})_{2} . .(\mathrm{ATA})_{8}$ & 234 & 54 & GF111410 \\
\hline $\begin{array}{l}\text { DVSSR_A020-R } \\
\text { DVSSR A021-F }\end{array}$ & $\begin{array}{l}\text { TTTCCTACTGAGCTGTAAAAGC } \\
\text { AAAGTGAGCCCAAAGTATAAGC }\end{array}$ & 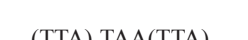 & 170 & 54 & 111 \\
\hline DVSSR_A021-R & TTTATTACTACGGTCGGCAAAC & (11A) & $1 / 0$ & 54 & GF111411 \\
\hline DVSSR_A022-F & TTTTAAAATAAAATCATCATCATCATCC & $(\mathrm{ATC})_{5} \mathrm{CTC}(\mathrm{ATC})_{2}$ & 150 & 53 & GF111412 \\
\hline DVSSR_A022-R & TATTTGTTACTTACATACAGATATGATG & & & & \\
\hline DVSSR_A023-F & AGTCCACTTTCGCTGTTGTG & $(\mathrm{TAT})_{9}$ & 141 & 56 & GF111413 \\
\hline DVSSR_A023-R & CATCACGGTCTGCATAAATCAC & & & & \\
\hline DVSSR_A024-F & TTCGACTTGTCGGCCTAATC & $(\mathrm{AAC})_{9}$ & 200 & 53 & GF111489 \\
\hline DVSSR_A024-R & TTTTACAGACAGTTTAGTGACG & & & & \\
\hline DVSSR_A025-F & GGTTCGCGTTCAGAATCG & $(\mathrm{ATT})_{8}$ & 159 & 53 & GF111414 \\
\hline DVSSR_A025-R & AACATTCGACTCTAGCAATACC & & & & \\
\hline DVSSR_A026-F & ACTGAATGTGTGCGTTTGTG & $(\mathrm{TAA})_{7} \mathrm{TT}(\mathrm{AT})_{8}$ & 243 & 54 & GF111415 \\
\hline DVSSR_A026-R & AAGACCCTTGGCGAATACAG & & & & \\
\hline $\begin{array}{l}\text { DVSSR_A027-F } \\
\text { DVSSR_A027-R }\end{array}$ & $\begin{array}{l}\text { TATCATAGCTTTCCACTTGAAC } \\
\text { TCCGAATTAAACAGCGTAGG }\end{array}$ & $(\mathrm{TTA})_{2} \mathrm{AA}(\mathrm{TTA})_{8}$ & 164 & 52 & GF111416 \\
\hline DVSSR_A028-F & TAATTTTGTAAAAGCCGTTTGG & $(\mathrm{ATA})_{2}(\mathrm{TAA})_{7}(\mathrm{TA})_{2}$ & 201 & 52 & GF111417 \\
\hline DVSSR_A028-R & ATTCCGAATAGGGAGTTTGAG & & & & \\
\hline
\end{tabular}

Continued on next page 


\begin{tabular}{|c|c|c|c|c|c|}
\hline Locus & Primer sequences $\left(5^{\prime}-3^{\prime}\right)$ & Repeats & Fragment size & $\mathrm{Ta}\left({ }^{\circ} \mathrm{C}\right)$ & $\begin{array}{c}\text { GenBank } \\
\text { accession No. }\end{array}$ \\
\hline DVSSR_A029-F & GACAGGTAATGAGGTGTGAGG & $(\mathrm{AAT})_{2} \ldots(\mathrm{ATA})_{9}$ & 144 & 55 & GF111418 \\
\hline DVSSR_A029-R & TTATGCTATGCGACGACGAC & & & & \\
\hline DVSSR_A030-F & TAGTTGTTCGGCGCAAGC & $(\mathrm{ACT})_{2} . .(\mathrm{ATT})_{10}$ & 159 & 54 & GF111419 \\
\hline DVSSR_A030-R & CAGCATACCATGTAATTTGTGG & & & & \\
\hline DVSSR_A031-F & TGTTGTTGTTGTTGTTGTGTTAG & $(\mathrm{GTT})_{3} . .(\mathrm{GTT})_{8}$ & 151 & 54 & GF111420 \\
\hline DVSSR_A031-R & CCGTTACCTATGTGCTATTGC & & & & \\
\hline DVSSR_A032-F & GGACAGGAGAGGAATACTTCG & $\begin{array}{c}(\mathrm{GTT})_{2} \mathrm{GTG}(\mathrm{GTT})_{3} \\
\ldots(\mathrm{GTT})_{2}\end{array}$ & 227 & 54 & GF111421 \\
\hline DVSSR_A032-R & GAGCAGCGGTACAAGGAG & & & & \\
\hline DVSSR_A033-F & TGGAGTCTTGAACAACTGATGG & $(\mathrm{GTT})_{4} \mathrm{GCT}(\mathrm{GTT})_{3}$ & 209 & 56 & GF111422 \\
\hline DVSSR_A033-R & ACAGCAACCATACGCAAGC & & & & \\
\hline DVSSR_A034-F & CCCTGTTATTGTGCCCTCTG & $(\mathrm{GTT})_{8}$ & 158 & 55 & GF111423 \\
\hline DVSSR_A034-R & TACCGTATGCGAGAGTAATGG & & & & \\
\hline DVSSR_A035-F & AAAAGGGCACAAATGGTTCG & $(\mathrm{AGC})_{5}(\mathrm{AGG})_{2}(\mathrm{AGC})_{2}$ & 200 & 55 & GF111424 \\
\hline DVSSR_A035-R & TGATATGCAACATTTCTCAGCTTG & & & & \\
\hline DVSSR_A037-F & TTTACGAGAAGAGTCTGTACCC & $\begin{array}{c}(\mathrm{GTA})_{3}(\mathrm{CAG})_{2}(\mathrm{TAG})_{5} . \\
(\mathrm{TAG})_{15}\end{array}$ & 237 & 54 & GF111425 \\
\hline DVSSR_A037-R & ACGACCACATCTACATTAAACC & & & & \\
\hline DVSSR_A038-F & CTAAAGGTACACACACGATTCG & $(\mathrm{AAT})_{11}$ & 219 & 54 & GF111426 \\
\hline DVSSR_A038-R & GGCGGAATAAATGAGAAAAGTG & & & & \\
\hline DVSSR_A039-F & ACTGTTGACTCCGCAGAGC & $(\mathrm{ATA})_{8}$ & 216 & 56 & GF111427 \\
\hline DVSSR_A039-R & CCACACGTATAGGTACACAAGC & & & & \\
\hline DVSSR_A040-F & ACTGCGATAATGCCACTGC & $(\mathrm{AAT})_{9} . .(\mathrm{GT})_{2}(\mathrm{AAT})_{3}$ & 236 & 55 & GF111428 \\
\hline DVSSR_A040-R & CGAGATAGCCTAGCGTATGTG & & & & \\
\hline DVSSR_A041-F & TTTTGGTCTCAGCATCTTTTCC & $\begin{array}{c}(\mathrm{GGT})_{5}(\mathrm{GGC})_{3} \\
\mathrm{GGA}(\mathrm{GGT})_{4}\end{array}$ & 153 & 54 & GF111429 \\
\hline DVSSR_A041-R & TTGTTACAGGCCATATTTACCC & & & & \\
\hline DVSSR_A042-F & CAGATGGCTGGAGGAATGG & $(\mathrm{ATT})_{7} . .(\mathrm{ATT})_{2}$ & 180 & 54 & GF111430 \\
\hline DVSSR_A042-R & TTCTATGGTGTAGGATGACGAG & & & & \\
\hline DVSSR_A043-F & ATTCAATGTACTATTTATTTCTTGGTTC & $\begin{array}{l}(\mathrm{ATT})_{8} . .(\mathrm{CT})_{2} . . \\
(\mathrm{CA})_{2} . .(\mathrm{ATT})_{2}\end{array}$ & 224 & 54 & GF111431 \\
\hline DVSSR_A043-R & TCAACAAAACAATTATCTATCAAAGTTC & & & & \\
\hline DVSSR_A044-F & CGGCTCGCTAACATATTGC & $(\text { TATT })_{6}$ & 144 & 54 & GF111490 \\
\hline DVSSR_A044-R & AAACTTACCTTGTGCAGCAC & & & & \\
\hline DVSSR_A045-F & CGTGGCGTTTTGAGAGTTAC & $(\mathrm{TACA})_{11}$ & 239 & 55 & GF111432 \\
\hline DVSSR_A045-R & ACGATAGTTACCATTGACAAGC & & & & \\
\hline DVSSR_A046-F & CACGACCGACCCGAGACG & $(\mathrm{CGTA})_{8}$ & 213 & 58 & GF111433 \\
\hline DVSSR_A046-R & TCGGAAAACGGCAGAGTCC & & & & \\
\hline DVSSR_A047-F & CCGCCCGCCTATAAATGTC & $(\mathrm{TATC})_{12}(\mathrm{TA})_{8}$ & 235 & 55 & GF111434 \\
\hline DVSSR_A047-R & GCGTTGCCCAGTAGAAGG & & & & \\
\hline DVSSR_A048-F & TGACGGCTGCTAACTCTACC & $(\mathrm{CTGC})_{6}(\mathrm{CAGC})_{3}$ & 158 & 57 & GF111435 \\
\hline DVSSR_A048-R & CCACGGTTGTGAGGAGTCG & & & & \\
\hline DVSSR_A049-F & TAGTGTTGCTGTCTTGTGTTG & $(\mathrm{TACA})_{4} \mathrm{TGCA}(\mathrm{TACA})_{4}$ & 217 & 54 & GF111436 \\
\hline DVSSR_A049-R & CGCAAATGGCTACCGTATC & & & & \\
\hline DVSSR_A050-F & CCATTGAATTGCGGTACTTCC & $(\mathrm{GTAT})_{6}$ & 161 & 55 & GF111437 \\
\hline DVSSR_A050-R & TGCGTTATGACAGTCTAGTCTC & & & & \\
\hline \multicolumn{6}{|c|}{48 SSR markers developed from biotype B } \\
\hline DVSSR_B001-F & GAGCTACAAAGATCTAGACAGG & $(\mathrm{TA})_{3} \mathrm{~T}(\mathrm{TA})_{10} \mathrm{~T}(\mathrm{TA})_{1}$ & 162 & 53 & GF111438 \\
\hline DVSSR_B001-R & CCGTGGAACTGTCAAACC & & & & \\
\hline DVSSR_B002-F & GCGGACAAACCAAATAATAACC & $\operatorname{GTAT}(\mathrm{GT})_{3} \mathrm{~T}(\mathrm{GT})_{8}$ & 191 & 54 & GF111439 \\
\hline DVSSR_B002-R & CGTCGTCTCGGATGAATCG & & & & \\
\hline DVSSR_B003-F & CCGCTGCTGGCAATACAC & $(\mathrm{TA})_{12}(\mathrm{AT})_{2} \mathrm{TT}(\mathrm{TA})_{4}$ & 191 & 55 & GF111440 \\
\hline DVSSR_B003-R & CATGCGTTGAGGAGGTAAGG & & & & \\
\hline DVSSR_B004-F & CACTATAATATGACAAAACTGGGTAATC & $(\mathrm{TA})_{10} \mathrm{TT}(\mathrm{TA})_{2}(\mathrm{AT})_{2}$ & 176 & 53 & GF111441 \\
\hline DVSSR_B004-R & GACCGACTTATGACAATGAACTG & & & & \\
\hline DVSSR_B005-F & GACAATGCACAAGAAGTAAACG & $(\mathrm{TA})_{11} \mathrm{~T}(\mathrm{TA})_{2} \mathrm{AG}(\mathrm{AT})_{2}$ & 145 & 54 & GF111442 \\
\hline DVSSR_B005-R & ATTACCACCAGAAGCCAGTC & & & & \\
\hline DVSSR_B006-F & GCTATGCGTATTCCGTAAGTCG & $(\mathrm{GT})_{10} \mathrm{GC}(\mathrm{GT})_{3}$ & 141 & 57 & GF111443 \\
\hline DVSSR_B006-R & GCTACCACCACAGACCTGAG & & & & \\
\hline DVSSR_B007-F & ATAACGCCACTGAAACATTGATG & $(\mathrm{TA})_{2} \mathrm{TT}(\mathrm{TA})_{10} \mathrm{TTATT}(\mathrm{TA})_{3}$ & 214 & 56 & GF111444 \\
\hline
\end{tabular}




\section{Table 1. Continued.}

\begin{tabular}{|c|c|c|c|c|c|}
\hline Locus & Primer sequences $\left(5^{\prime}-3^{\prime}\right)$ & Repeats & Fragment size & $\mathrm{Ta}\left({ }^{\circ} \mathrm{C}\right)$ & $\begin{array}{c}\text { GenBank } \\
\text { accession No. }\end{array}$ \\
\hline DVSSR_B007-R & GCAACAGATATGAATACAGAGTAGC & & & & \\
\hline DVSSR_B008-F & GCGTTACGAAGATGTGTGTC & $\operatorname{ATA}(\mathrm{AT})_{9} \ldots(\mathrm{AT})_{3} \ldots(\mathrm{AT})_{3}$ & 239 & 55 & GF111445 \\
\hline DVSSR B008-R & GTTCCTCCGGCCTTCCAC & & & & \\
\hline DVSSR_B009-F & CGTGTGCCGTTCAAGGTC & TGTA(TG) $)_{2}$ TA(TG) $)_{11}$ & 235 & 56 & GF111446 \\
\hline DVSSR_B009-R & CCCCGCCGTTCATCAGAC & & & & \\
\hline DVSSR_B010-F & AGACTGTCGTAACGCATTCAC & $(\mathrm{TA})_{14}$ & 223 & 56 & GF111447 \\
\hline DVSSR_B010-R & GGCTGATAAAGGTGGCACTAG & & & & \\
\hline DVSSR_B011-F & TACAGGATACAATATTCACACTCAG & $(\mathrm{AC})_{14}$ & 166 & 54 & GF111448 \\
\hline DVSSR_B011-R & GTACAAACATATGATCTCGATTCG & & & & \\
\hline DVSSR_B012-F & TCAGCACGAGTCTATTGAAACG & $\begin{array}{c}(\mathrm{GT})_{6} . . \mathrm{TT}(\mathrm{GT})_{2} \mathrm{TT} \\
(\mathrm{GT})_{3} \mathrm{TT}(\mathrm{GT})_{6}\end{array}$ & 228 & 56 & GF111449 \\
\hline DVSSR_B012-R & AGCGACGGTGATAATAAAGTGG & & & & \\
\hline DVSSR_B013-F & ATATTAAGTTCCTATGTTTCCTTACC & $(\mathrm{AT})_{14} . .(\mathrm{AT})_{2}$ & 232 & 54 & GF111450 \\
\hline DVSSR_B013-R & ACATCTACAATTATAGAACACACAAC & & & & \\
\hline DVSSR_B014-F & CACCTGTGTCTGGAAATATACC & $(\mathrm{AT})_{9}$ & 183 & 54 & GF111451 \\
\hline DVSSR_B014-R & CCACATCATAGGTCAGTATTGC & & & & \\
\hline DVSSR_B015-F & TCTAAACAGCCCCTGAAATTAAAC & $(\mathrm{AT})_{10} \mathrm{AATT}(\mathrm{AT})_{3}$ & 187 & 55 & GF111452 \\
\hline DVSSR_B015-R & AGCTCACACTTGTATTTATTTCATTG & & & & \\
\hline DVSSR_B016-F & ATGGTCCAACAGGTCTTAGTG & $(\mathrm{TA})_{2} . .(\mathrm{TA})_{2} . .(\mathrm{TA})_{12}$ & 200 & 55 & GF111453 \\
\hline DVSSR_B016-R & AATCGATGTGCTACTATGAACG & & & & \\
\hline DVSSR_B017-F & AATACCACCCGCATGTAATG & $(\mathrm{TA})_{4} \mathrm{~A}(\mathrm{TA})_{2} \mathrm{~A}(\mathrm{TA})_{5} \mathrm{~T}(\mathrm{TA})_{3}$ & 177 & 53 & GF111454 \\
\hline DVSSR_B017-R & ATAGTAAGGCGACATAAGTACG & & & & \\
\hline DVSSR_B018-F & ATGGACGTACTTCAAGAATAGC & $\begin{array}{l}(\mathrm{CT})_{4}(\mathrm{AT})_{4} . .(\mathrm{CT})_{4} \\
(\mathrm{AT})_{3} \mathrm{AA}(\mathrm{AT})_{7}\end{array}$ & 238 & 53 & GF111455 \\
\hline DVSSR_B018-R & ACATTGTTTTATAGGACCAACG & & & & \\
\hline DVSSR_B019-F & AAGATAATAAATGGCGGAGTAACAC & $(\mathrm{AT})_{5} \mathrm{~A}(\mathrm{AT})_{10} . .(\mathrm{AT})_{7}$ & 180 & 56 & GF111456 \\
\hline DVSSR_B019-R & ATACGCATTCGGCTCAACAC & & & & \\
\hline DVSSR_B020-F & CACATATCGGAATGTAATTTTAGTAC & $(\mathrm{AT})_{3} . .(\mathrm{AT})_{12}$ & 238 & 53 & GF111457 \\
\hline DVSSR_B020-R & GACTACCTTACAGAGAATAGACC & & & & \\
\hline DVSSR_B021-F & AGGTTATTGGTCAGTGGTGTG & $(\mathrm{AT})_{15}$ & 168 & 55 & GF111458 \\
\hline DVSSR_B021-R & TGAAGTGTCTTCCGCATCG & & & & \\
\hline DVSSR_B022-F & GTTTTGTGTTGTATGTTTATATTTCAGG & $(\mathrm{AT})_{14}$ & 137 & 56 & GF111459 \\
\hline DVSSR_B022-R & GCACTTAGACAATAAATACTAAAGAAGC & & & & \\
\hline DVSSR_B023-F & GCTTGAACGACGAACTCATC & $(\mathrm{AT})_{12}$ & 204 & 55 & GF111460 \\
\hline DVSSR_B023-R & AAAACAAACCTCCСCTCTGC & & & & \\
\hline DVSSR_B024-F & CAACTACCAGTTTGTACTCAAG & $(\mathrm{AT})_{2} \mathrm{AA}(\mathrm{AT})_{4} . .(\mathrm{AT})_{13}$ & 250 & 53 & GF111461 \\
\hline DVSSR_B024-R & ACACATGTCCAAAATGTCAATC & & & & \\
\hline DVSSR_B025-F & CGTTCGCCCACTACAGGTAC & $(\mathrm{TG})_{15}$ & 154 & 57 & GF111462 \\
\hline DVSSR_B025-R & TTCGTCGCCAACCCAACC & & & & \\
\hline DVSSR_B026-F & AGGGCACACCAACAGTCC & $(\mathrm{AC})_{9} \mathrm{AT}(\mathrm{AC})_{2}$ & 163 & 56 & GF111463 \\
\hline DVSSR_B026-R & GTCCAGTGCAACGCTAAGG & & & & \\
\hline DVSSR_B027-F & ACAGAGCCTTTACTTACAAACC & $(\mathrm{ACAT})_{2}(\mathrm{AC})_{12}$ & 193 & 54 & GF111464 \\
\hline DVSSR_B027-R & TCAGCCGTGTAATACAATTAGG & & & & \\
\hline DVSSR_B028-F & GAACGACCGATGTGTATTGC & $(\mathrm{TG})_{12}$ & 234 & 55 & GF111465 \\
\hline DVSSR_B028-R & TGTGTTGCGACCAGTGTAC & & & & \\
\hline DVSSR_B029-F & CTACACGCCATAAGAACCATAGG & $(\mathrm{TAT})_{9}$ & 224 & 56 & GF111466 \\
\hline DVSSR_B029-R & ATGAACGCCTAGTTAACAGTGG & & & & \\
\hline DVSSR_B030-F & AATTCAGCCTATCTTATGTGTCG & $(\mathrm{TTA})_{2}(\mathrm{TAA})_{2}(\mathrm{ATT})_{8}$ & 240 & 54 & GF111467 \\
\hline DVSSR_B030-R & TAATTTCAGTTAAAGATGGACTAGAG & & & & \\
\hline DVSSR_B031-F & TGCTTATTAGACATACATATTATCGC & $(\mathrm{TA})_{3}(\mathrm{ATA})_{2} \ldots(\mathrm{ATT})_{8}$ & 183 & 54 & GF111468 \\
\hline DVSSR_B031-R & AACACAATAGCTCAGAGATTTACC & & & & \\
\hline DVSSR_B032-F & TTTATTTTCGACCGATCTCACC & $\begin{array}{c}(\mathrm{ATT})_{9} . .(\mathrm{ATC})_{2} \mathrm{AGT} \\
(\mathrm{ATT})_{3}\end{array}$ & 196 & 54 & GF111469 \\
\hline DVSSR_B032-R & TTGGACTATCTACCCTACATGC & & & & \\
\hline DVSSR_B033-F & AGCCATACCATGAAAGTGTACC & $(\mathrm{ACT})_{8}$ & 148 & 55 & GF111470 \\
\hline DVSSR_B033-R & GAACGGAGTCGAGGAAGAATC & & & & \\
\hline DVSSR_B034-F & AGTTGTATTTAGTTTGTAAGTGTACG & $(\mathrm{TAT})_{9}$ & 143 & 55 & GF111491 \\
\hline DVSSR_B034-R & TTTTGCCACGACGACCTC & & & & \\
\hline DVSSR_B035-F & CACTTCAACCTACAGAATTGTTTGC & $(\mathrm{TTA})_{8}$ & 185 & 57 & GF111471 \\
\hline DVSSR_B035-R & GCGTGGTGGACATTGATATTGG & & & & \\
\hline
\end{tabular}

Continued on next page 


\begin{tabular}{|c|c|c|c|c|c|}
\hline Locus & Primer sequences $\left(5^{\prime}-3^{\prime}\right)$ & Repeats & Fragment size & $\mathrm{Ta}\left({ }^{\circ} \mathrm{C}\right)$ & $\begin{array}{c}\text { GenBank } \\
\text { accession No. }\end{array}$ \\
\hline $\begin{array}{l}\text { DVSSR_B036-F } \\
\text { DVSSR_B036-R }\end{array}$ & $\begin{array}{l}\text { AAATTAAGTCTGAACAGGTAAATCC } \\
\text { TTCTTTGCGTCTTTGATCTGG }\end{array}$ & $(\mathrm{GCA})_{2}(\mathrm{ACA})_{8}$ & 200 & 54 & GF111472 \\
\hline DVSSR_B037-F & CATTCGCCACAGCAACAAC & $(\mathrm{CAA})_{8}$ & 215 & 55 & GF111473 \\
\hline DVSSR_B037-R & ATGGTATCGTCGTCGTAATCG & & & & \\
\hline DVSSR_B038-F & AATACCATCGTCCCATAAGAGC & $(\mathrm{ATT})_{9}$ & 184 & 56 & GF111474 \\
\hline DVSSR_B038-R & CGTGATCCGACTACTGTGTAAC & & & & \\
\hline DVSSR_B039-F & CGGCGTGTAACTTTGATTGG & $(\mathrm{TAC})_{9}$ & 167 & 54 & GF111475 \\
\hline DVSSR_B039-R & GCGGTTCACATTTCATTATTCC & & & & \\
\hline DVSSR_B040-F & AACTTGTGGTGGTTGTATTGC & $\begin{array}{l}(\mathrm{CTG})_{2} \mathrm{CCT}(\mathrm{CTT})_{8} . \cdot \\
(\mathrm{CTT})_{2} . .(\mathrm{CTT})_{2}\end{array}$ & 162 & 55 & GF111476 \\
\hline DVSSR_B040-R & GAATCTGATACTGCTGCTGAAG & & & & \\
\hline DVSSR_B041-F & GGAGAATAACTACAAGCAGAGC & $(\mathrm{ACG})_{8}$ & 208 & 55 & GF111477 \\
\hline DVSSR_B041-R & ACGAAGGGCGACAACAAC & & & & \\
\hline DVSSR_B042-F & GCTGAGAGATTTAACGGAACC & $(\mathrm{GAG})_{7}$ & 160 & 54 & GF111478 \\
\hline DVSSR_B042-R & ACCACCAATCGCAGTTACC & & & & \\
\hline DVSSR_B043-F & GGGATGGCATAATGGATTTGG & $(\mathrm{ATA})_{9}$ & 181 & 54 & GF111479 \\
\hline DVSSR_B043-R & TTCGTCTGGTTGGTGAAGG & & & & \\
\hline DVSSR_B044-F & GTAAACGACGACAACACAGC & $(\mathrm{AAT})_{2} \mathrm{GAT}(\mathrm{AAT})_{7}$ & 189 & 55 & GF111480 \\
\hline DVSSR_B044-R & CAGGATAACAGCAGAATACACG & & & & \\
\hline DVSSR_B045-F & TTCCTCGATCTGCTCCTTGG & $\begin{array}{c}(\mathrm{CTC})_{3} \mathrm{TT}(\mathrm{CTT})_{3}(\mathrm{CCT})_{3} \\
\mathrm{CTT}(\mathrm{CCT})_{2}\end{array}$ & 164 & 57 & GF111481 \\
\hline DVSSR_B045-R & GCGATTGAAGTTGATACGAATTGG & & & & \\
\hline DVSSR_B046-F & ATGACAAGAAAGACAAACAATG & $(\text { ATTTA })_{6}$ GTTT(ATTT) $)_{2}$ & 232 & 52 & GF111482 \\
\hline DVSSR_B046-R & GGCTTGTGTTAAAATAATCACC & & & & \\
\hline DVSSR_B047-F & GGCTCCGATTGGTTGTTCC & $(\mathrm{ACAT})_{5} \mathrm{TT}(\mathrm{AT})_{7}$ & 200 & 56 & GF111483 \\
\hline DVSSR_B047-R & GCGGTGTAGTAATGACGAAGG & & & & \\
\hline DVSSR_B048-F & CATCGAGATTAATAAGTAGTTAGGG & $(\text { GTTGG })_{3}$ & 229 & 53 & GF111484 \\
\hline DVSSR_B048-R & ATTTAATAGTCATATACCAACAACCC & & & & \\
\hline \multicolumn{6}{|c|}{15 SSR markers developed from the shared loci of biotype A and biotype B } \\
\hline DVSSR_AB001-F & GTAATGTTTTTGCTGGATCTAATA & $(\mathrm{AT})_{8} \mathrm{~T}(\mathrm{AT})_{2} \mathrm{C}(\mathrm{AT})_{3}$ & 166 & 53 & GF111485 \\
\hline DVSSR_AB001-R & GGGCTCTAGGTTGTCCGATT & & & & \\
\hline DVSSR_AB002-F & TTTTGTGCGGCACGGTACTC & $(\mathrm{AT})_{12}$ & 153 & 56 & GF111492 \\
\hline DVSSR_AB002-R & GGTAATGATGAACACCACACA & & & & \\
\hline DVSSR_AB005-F & AGTTTATTGTGTCTGAAACGCA & $\mathrm{T}_{6} \mathrm{~A}_{4} \mathrm{TA} . . \mathrm{T}_{4} . .(\mathrm{GT})_{3}$ & 172 & 57 & GF111486 \\
\hline DVSSR_AB005-R & AACCCAACACAAGGGGGTCG & & & & \\
\hline DVSSR_AB008-F & GAGTATCACCGTAAAGTGAC & $\left(\right.$ TTAAAA $_{6}$ & 185 & 50 & GF111502 \\
\hline DVSSR_AB008-R & CTGTCTTATTTTTATTTGACAATC & & & & \\
\hline DVSSR_AB009-F & GTTACCAACCTTTATTATCATTG & $(\mathrm{AT})_{8} . .(\mathrm{CA})_{3}$ & 180 & 52 & GF111493 \\
\hline DVSSR_AB009-R & TGCTCACACACACACCTTACT & & & & \\
\hline DVSSR_AB010-F & GAGGTGTTTCACCTACACAGT & $(\mathrm{AC})_{11} \mathrm{GT}(\mathrm{AC})_{3} \mathrm{GC}(\mathrm{AC})_{3}$ & 189 & 52 & GF111487 \\
\hline DVSSR_AB010-R & GAGTATGTGTTCAATAACTCG & & & & \\
\hline DVSSR_AB014-F & CTTTTGCTATCGGACGGCCC & $(\mathrm{GGT})_{5} . .(\mathrm{GGC})_{4} . .(\mathrm{GGT})_{6}$ & 180 & 59 & GF111488 \\
\hline DVSSR_AB014-R & TGCGCTAGTTCCATCGACGTA & & & & \\
\hline DVSSR_AB018F & CTGTGCTTTGCCACAGTAATA & $(\mathrm{ATTACT})_{2}(\mathrm{ATT})_{4}$ & 211 & 53 & GF111494 \\
\hline DVSSR_AB018R & CCAACGCGTATAATACAGGTA & & & & \\
\hline DVSSR_AB019F & TCCAACTATCGCACTCCTTGC & $\begin{array}{c}(\mathrm{TA})_{3}(\mathrm{TTAA})_{3} \mathrm{GT} \\
(\mathrm{ATT})_{3} . .(\mathrm{AT})_{3}\end{array}$ & 289 & 54 & GF111495 \\
\hline DVSSR_AB019R & TCTGAAAATCGATCGCGACCC & & & & \\
\hline DVSSR_AB020F & GCATTACTTGTAAACCGAGCC & $(\mathrm{TAG})_{12}$ & 215 & 54 & GF111496 \\
\hline DVSSR_AB020R & CAAAAGTCATAAGCGTTGTGC & & & & \\
\hline DVSSR_AB021F & ACTGTGTGCATGGAGAACCC & $\begin{array}{c}(\text { TAAA })_{2} . .(\text { TATAAA })_{2} \\
. .(\text { TA })_{2}\end{array}$ & 207 & 57 & GF111497 \\
\hline DVSSR_AB021R & TTGATACTTCGGGACGGGTG & & & & \\
\hline DVSSR_AB022F & ACGCCCATTAGGGCAAACAG & $(\mathrm{AT})_{3} . .(\mathrm{AT})_{6}$ & 293 & 57 & GF111498 \\
\hline DVSSR_AB022R & CTCTCCTGTAAATCGCATGCT & & & & \\
\hline DVSSR_AB023F & GCGCAGCATATTCGCAAATGT & $(\mathrm{AAT})_{9}$ & 213 & 58 & GF111499 \\
\hline DVSSR_AB023R & TCATCTCGGAGACCACCGAAA & & & & \\
\hline DVSSR_AB024F & CAACCGAACTCTTCAATCACC & $(\text { TAATA })_{2} . .(\text { TAATA })_{4}$ & 286 & 52 & GF111500 \\
\hline DVSSR_AB024R & AATGTGATACTCGCAACAC & & & & \\
\hline DVSSR_AB025F & GCTAACCAATACATCTTGTTC & $(\mathrm{TA})_{3} . .(\mathrm{TAA})_{4}$ & 237 & 52 & GF111501 \\
\hline DVSSR_AB025R & CGTAGAGATCGTTCATTGCCA & & & & \\
\hline
\end{tabular}

$\mathrm{Ta}=$ annealing temperature of the primer pairs. 


\section{ACKNOWLEDGMENTS}

We thank Karl Lund of UCD for providing phylloxera samples. Research supported in part by the Viticulture Consortium West. Trade names or commercial products in this publication are mentioned solely for the purpose of providing specific information, and does not imply recommendation or endorsement by the United States Department of Agriculture.

\section{REFERENCES}

Benson G (1999). Tandem repeats finder: a program to analyze DNA sequences. Nucleic Acids Res. 27: 573-580.

Corrie AM, Crozier RH, Van Heeswijck R and Hoffmann AA (2002). Clonal reproduction and population genetic structure of grape phylloxera, Daktulosphaira vitifoliae, in Australia. Heredity 88: 203-211.

Granett J, Walker A, De Benedictis J, Fong G, et al. (1996). California grape phylloxera more variable than expected. Calif Agric. $50: 9-13$.

Granett J, Walker MA, Kocsis L and Omer AD (2001). Biology and management of grape phylloxera. Annu. Rev. Entomol. 46: 387-412.

Lin H and Walker MA (1996). Extraction of DNA from a single egg of grape phylloxera (Daktulosphaira vitifoliae Fitch) for use in RAPD testing. Vitis 35: 87-89.

Lin H, Walker MA, Hu R and Granett J (2006) New simple sequence repeat loci for the study of grape phylloxera (Daktulosphaira vitifoliae) genetics and host adaptation. Am. J. Enol. Vitic. 57: 33-40.

Skinkis P, Walton V and Kaiser C (1995). Grape Phylloxera: Biology and Management in the Pacific Northwest. Oregon State University, Extension Service EC 1463-4. Available at [http://extension.oregonstate.edu/catalog/pdf/ec/ ec1463-e.pdf]. Accessed June 9, 2012.

Wapshere AJ and Helm KF (1987). Phylloxera and Vitis: an experimentally testable co-evolutionary hypothesis. Am. J. Enol. Vitic. 38: 16-22. 